\title{
PACE: A Test Bed for the Dynamics and Control of Flexible Multibody Systems
}

\author{
Moon K. Kwak, Monty J. Smith and Alok Das \\ The USAF Phillips Laboratory \\ OLAC PL/VTSS \\ Edwards AFB, CA 93523
}

\begin{abstract}
The Phillips Laboratory at Edwards AFB has constructed a test bed for the validation and comparison of modeling and control theories for the dynamics and control of flexible multibody systems. This project is called the Planar Articulating Controls Experiment (PACE). This paper presents the experimental apparatus for PACE and the problem formulation. An in depth analysis on DC motor dynamics was also performed.
\end{abstract}

\section{Introduction}

The Air Force remains active in space systems, and hardware, such as space robots, rotorcraft and spacecraft, consists of subsystems which can be described as flexible multibodies. The dynamics and control of flexible multibody systems has been of interest for many years. (Refs. 1-11). Identifying, modeling and controlling such systems using various theories with confidence has also become an important issue. At present, a real need exists for the validation and comparison of various modeling and control theories based on an actual hardware experiment. However, compared to theoretical developments and number of computer programs available, experimental verification has never been conducted. To this end, the Phillips Laboratory at Edwards AFB has constructed a flexible multibody structure which consists of 2 flexible beams connected in series with motors at both the hub and the elbow joint. Figure 1 shows the multibody body structure named the Planar Articulating Controls Experiment (PACE).

\section{Experimental Apparatus}

Figure 2 shows the PACE control loop which includes 2 flexible arm manipulator, various sensors and actuators, amplifiers, an AC-100 data acquisition controller and the VAX workstation. Actuators and sensors are connected to D/A and A/D interfaces of the AC-100 controller through amplifiers. Sixteen channcls are available on each D/A and A/D interfaces. The VAX 3100 workstation controls the AC-100 data acquisition controller with the ISI real-time monitor software which includes MatrixX, Autocode and Interactive Animation module.

The motion of the multibody test article is constrained to horizontal motion on a 14 foot square granite table. An air compressor is used to activate an air bearing, which allows the whole system to float on air so that friction forces do not exist between the support plate and the granite table. The shoulder motor is fixed at the center of the granite slab and the elbow motor is located at the junction of two beams. Thus, the arms can be driven 
through some trajectory using the DC motors.

Sensors for PACE include:

1. Encoders count the change of a square wave; its resolution is 2540 cycles per revolution. A differential interface converts the encoder output to the number of changes in the data acquisition time interval, which is proportional to the angular velocity of the motor shafts. Tachometers are not being used currently due to their inaccuracy in measurements, as reported by the manufacturer, PMI company.

2. Lasers trace the positions of two arms. JPL is designing and testing the hardware implementation.

3. Accelerometers will measure accelerations of arbitrary points of interest on the structure.

4. Piezoceramic Sensors will measure strains along beams.

Actuators for PACE include:

1. PMI DC motors provide primary torques to slew the arms. Both motors have a gear box.

2. Piezoceramic actuators will suppress vibrations of the arms.

3. Reaction wheels (Optional) will apply a secondary moment to suppress vibrations of the arms.

The free body diagram for PACE, seen in Fig. 3, can be divided into 4 components; 2 DC motors and 2 nexible beams with lip masses at both ends. The dynamics of DC motors should be incorporated into the dynamics of the whole system. Modeling of the PMI DC motor with a gearbox is not an easy task since the gear box of DC motor causes noise, backlash and power loss.

\section{Problem Formulation}

Before the integration of the components of PACE, DC motor identification was performed using the unit-step and sine-sweep response tests. The sine-sweep response of the elbow, shown in Fig. 5, illustrates the dominant effect of static friction torque on the system response. Based on these tests, the mathematical model includes mass moment of inertia, viscous and Coulomb damping and static friction. Thus, the equation of motion for the shoulder motor is given as

$$
n^{2} I_{s m} \ddot{\theta}_{1}+C_{s v} \dot{\theta}_{1}+C_{s c} s g n\left(\dot{\theta}_{1}\right)=n\left(T_{s}-T_{s f}\right)-T_{1}
$$

while the elbow motor is described as

$$
n^{2} I_{e m} \ddot{\theta}_{2}+C_{e v} \dot{\theta}_{2}+C_{e c} \operatorname{sgn}\left(\dot{\theta}_{2}\right)=n\left(T_{e}-T_{e f}\right)-T_{2}
$$


where $n$ is the gear reduction ratio, $I_{s m}$ and $I_{e m}$ are the mass moment of inertias of the shoulder and elbow motors, respectively, $\theta_{1}$ and $\theta_{2}$ are the angular displacements of each motor relative to motor housings, $C_{s v}$ and $C_{e v}$ represent the viscous damping coefficients of each motor, $C_{s c}$ and $C_{e c}$ represent the Coulomb frictions of each motor, $T_{s}$ and $T_{e}$ are the motor torques, $T_{s f}$ and $T_{e f}$ represent the static friction torques of each motor, and $T_{1}$ and $T_{2}$ represent the torques transferred to each arm of the shoulder and the forearm, respectively. Note that the subscripts $s$ and $e$ denote the shoulder motor and the elbow motor, respectively. The static friction torques, $T_{s f}$ and $T_{e f}$, are expressed in terms of the motor torques, $T_{s}$ and $T_{e}$.

$$
\begin{aligned}
& T_{s f}= \begin{cases}-T_{s f}^{*} & \text { if } T_{s}<-T_{s f}^{*} ; \\
T_{s} & \text { if }-T_{s f}^{*} \leq T_{s} \leq T_{s f}^{*} ; \\
T_{s f}^{*} & \text { if } T_{s}>T_{s f}^{*} .\end{cases} \\
& T_{e f}= \begin{cases}-T_{e f}^{*} & \text { if } T_{e}<-T_{e f}^{*} ; \\
T_{\epsilon} & \text { if }-T_{e f}^{*} \leq T_{e} \leq T_{e f}^{*} ; \\
T_{e f}^{*} & \text { if } T_{e}>T_{e f}^{*} .\end{cases}
\end{aligned}
$$

Based on the circuits of the DC motors and the servo amplifiers, we can express the motor torque in terms of the input voltage to the servo amplifier

$$
\begin{gathered}
T_{s}=K_{s T}\left(\alpha_{s} V_{s}+\beta_{s}\right) \\
T_{c}=-K_{e} T\left(\alpha_{e} V_{e}+\beta_{e}\right)
\end{gathered}
$$

where $K_{s T}$ and $K_{e T}$ are motor constants, $\alpha_{s}, \alpha_{e}$ and $\beta_{s}$ and $\beta_{e}$ are constants of servo amplifiers, and $V_{s}$ and $V_{e}$ are the input voltage to the servo amplifiers, respectively. Equations (3) and (4) relate the output voltages of the $\mathrm{AC}-100$ controller to the motor torques of DC motors. Note that the minus sign is added in Eq. (4) because of the direction. Parameters of the PMI DC motors were identified independently due to errors in the manufacturer data. The identification results for the shoulder and elbow motors are tabulated in Tables 1 and 2. The material properties of each arm, listed in Fig. 5, will be identified in the near future.

Equations of motion for PACE are derived using the Lagrangian approach (Ref. 12). Since each beam is represented by a distributed parameter system, the resulting equations have the form of hybrid equations, i.e., the combination of ordinary differential equations and partial differential equations. For the numerical simulation and control design, the elastic displacements are discretized with the assumed-mode technique, resulting in highorder nonlinear ordinary differential equations. Thus, recalling Eqs. (1) and (2), we may write the following state equation for the whole system

$$
\dot{\mathbf{x}}=\mathbf{f}(\mathbf{x}, \mathbf{F})
$$

where

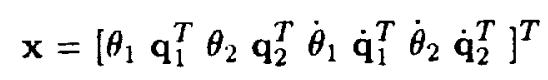

and

$$
\mathbf{F}=\left[V_{s} \mathbf{v}_{1}^{T} V_{e} \mathbf{v}_{2}^{T}\right]^{T}
$$


in which $\mathbf{x}$ is the state, $\mathbf{F}$ is the control vector, $\mathbf{q}_{1}$ and $\mathbf{q}_{2}$ represent the generalized coordinates for the elastic vibration of each arm, and $\mathbf{v}_{1}$ and $\mathbf{v}_{2}$ are the input voltage to the amplifiers of piezoceramic actuators and reaction wheels mounted on each beam, respectively.

The nonlinear sensor equations are

$$
\mathbf{y}=\mathbf{g}(\mathbf{x}, \dot{\mathbf{x}})
$$

where $\mathbf{y}$ consists of the encoder outputs, output voltages of piezoceramic sensors and accelerometers.

The dynamic analysis of PACE will be performed using both commercial and in-house algorithms.

\section{Control Design}

The VAX workstation is equipped with integrated software for data acquisition and control, the Real-Time Monitor Control. Fortran or C subroutines can be incorporated into the real-time control of PACE. The control objectives of PACE are to drive the arms to a certain position with a specified line of sight in minimum time and to suppress vibrations simultaneously based on sensor measurements. The authors are performing a survey of possible control techniques such as neural network control, nonlinear control and adaptive control techniques that can be applied to PACE. The difficulty of finding a control algorithm for PACE is in the inherent nonlinearity of the system, including the structural, sensor and actuator dynamics.

\section{Summary and Conclusions}

The experimental apparatus for PACE has been described in detail. The current configuration enables us to verify the numerical results of modeling and control theories for flexible multibody systems experimentally. Presently, both sensors and actuators are still being mounted to the PACE structure, and system identifications of its components must continue to be performed. However, in the near future, PACE will be the ideal test bed for researchers studying the dynamics and control of flexible multibody systems.

\section{References}

1. Meirovitch, L. and IIale, A. L., "Synthesis and Dynamic Characteristics of Large Structures with Rotating Substructures," Proceedings of the IUTAM Symposium on the Dynamics of Multi-Body Systems, (Editor: K. Magnus), Springer-Verlag, Berlin, 1978, pp. 231-244.

2. Hollerbach, J. M., "A Recursive Lagrangian Formulation of Manipulator Dynamics and a Comparative Study of Dynamics Formulation Complexity," IEEE Transactions on Systems, Man, and Cylernctics, Vol. SMC-10, No. 11, 1980, pp. 730-736. 
3. Huston, R. L., "Flexibility Effects in Multibody System Dynamics," Mechanics Research Communications, Vol. 7, No. 4, 1980, pp. 261-268.

4. Huston, R. L., "Multi-Body Dynamics Including the Effects of Flexibility and Compliance," Computers and Structures, Vol. 14, No. 5-6, 1981, pp. 443-451.

5. Book, W. J., "Recursive Lagrangian Dynamics of Flexible Manipulator Arms," The International Journal of Robotics Research, Vol. 3, No. 3, 1984, pp. 87-101.

6. Yoo, W. S. and Haug, E. J., "Dynamics of Articulated Structures. Part I. Theory," Journal of Structural Mechanics, Vol. 14, No. 1, 1986, pp. 105-126.

7. Yoo, W. S. and Haug, E. J., "Dynamics of Articulated Structures. Part II. Computer Implementation and Applications," Journal of Structuml Mechanics, Vol. 14, No. 2, 1986, pp. 177-189.

8. Changizi, K. and Shabana, A. A., "A Recursive Formulation for the Dynamic Analysis of Open Loop Deformable Multibody Systems," Journal of Applied Mechanics, Vol. 55, 1988, pp. 687-693.

9. Shabana, A. A., "On the Use of the Finite Element Method and Classical Approximation Techniques in the Non-linear Dynamics of Multibody Systems," International Journal of Non-Linear Mechanics, Vol. 25, No. 2, 1990, pp. 153-162.

10. Meirovitch, L. and Kwak, M. K., "Rayleigh-Ritz Based Substructure Synthesis for Flexible Multibody Systems," AIAA Journal, Vol. 29, No. 10, Oct. 1991, pp. 1709-1719.

11. Meirovitch, L. Stemple, T. and Kwak, M. K., "Dynamics and Control of Flexible Space Robots," presented at the Symposium on Mechanical Systems with Time-Varying Topology, CSME Mechanical Engineering Forum 1990, June 3-9, 1990, Toronto, Canada.

12. Kwak, M. K. and Meirovitch, L. "A New Approach to the Maneuvering and Control of Flexible Multibody Systems," Journal of Guidance, Control and Dynamics (to appear). 


\begin{tabular}{|c|c|c|}
\hline & Shoulder Motor & Unit \\
\hline$n$ & 60 & \\
\hline$I_{s m}$ & $1.043 \times 10^{-4}$ & $\mathrm{~kg} \mathrm{~m}$ \\
\hline$C_{s v}$ & 0.829 & $\mathrm{~N} \mathrm{~m} / \mathrm{rad} / \mathrm{s}$ \\
\hline$C_{s c}$ & 1.097 & $\mathrm{~N} \mathrm{~m}$ \\
\hline$K_{s T}$ & 0.084 & $\mathrm{~N} \mathrm{~m} / \mathrm{A}$ \\
\hline$\alpha_{s}$ & 1.7189 & $A / V$ \\
\hline$\beta_{s}$ & 0.1590 & $A$ \\
\hline$T_{s}^{*}$ & 0.030 & $N \mathrm{~m}$ \\
\hline
\end{tabular}

Table 1. Data for the Shoulder Motor (PMI S9M4II)

\begin{tabular}{|c|c|c|}
\hline & Elbow Motor & Unit \\
\hline$n$ & 60 & \\
\hline$I_{e m}$ & $0.278 \times 10^{-4}$ & $\mathrm{~kg} \mathrm{~m}^{2}$ \\
\hline$C_{e v}$ & 0.073 & $N \mathrm{~m} / \mathrm{rad} / \mathrm{s}$ \\
\hline$C_{e c}$ & 0.423 & $N \mathrm{~m}$ \\
\hline$K_{e T}$ & 0.030 & $N \mathrm{~m} / \mathrm{A}$ \\
\hline$\alpha_{e}$ & 1.5479 & $\mathrm{~A} / \mathrm{V}$ \\
\hline$\beta_{e}$ & 0.0289 & $\mathrm{~A}$ \\
\hline$T_{e f}^{*}$ & 0.012 & $N \mathrm{~m}$ \\
\hline
\end{tabular}

Table 2. Data for the Elbow Motor (PMI S6M4H) 


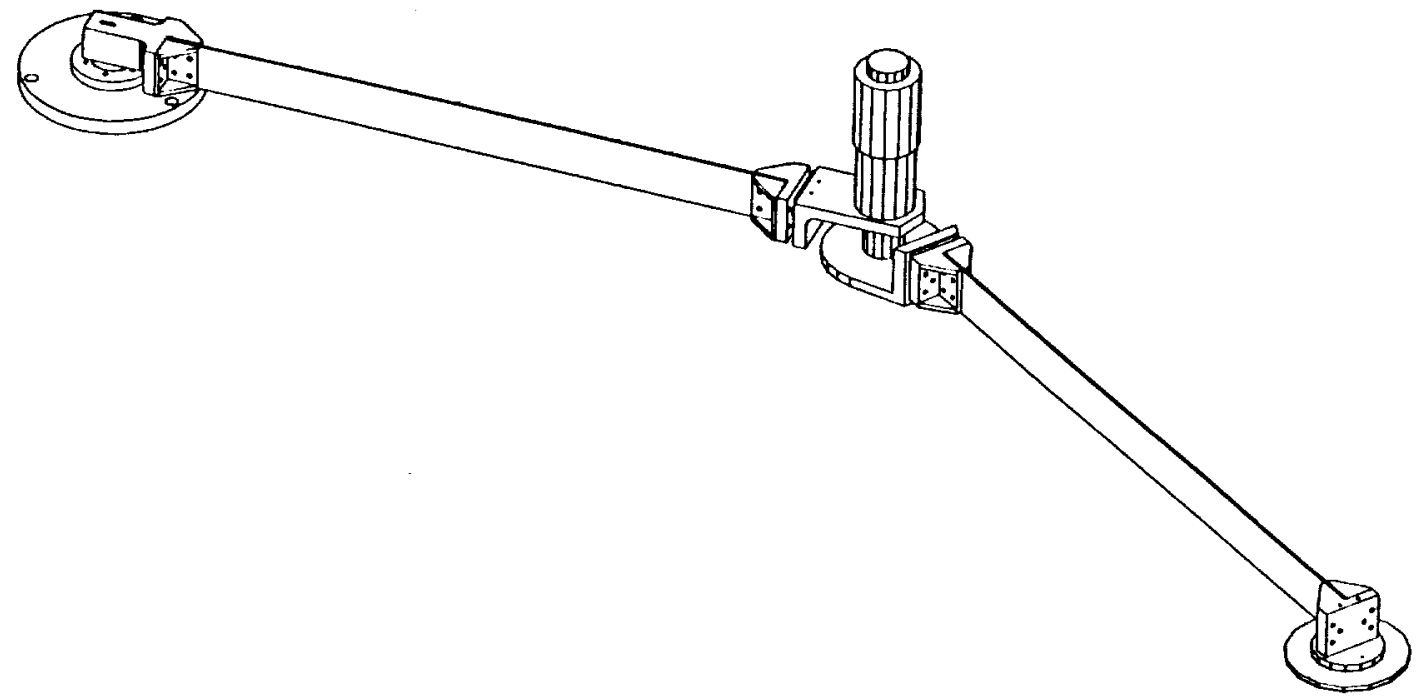

Fig. 1. The Planar Articulating Controls Experiment (PACE) Test Article

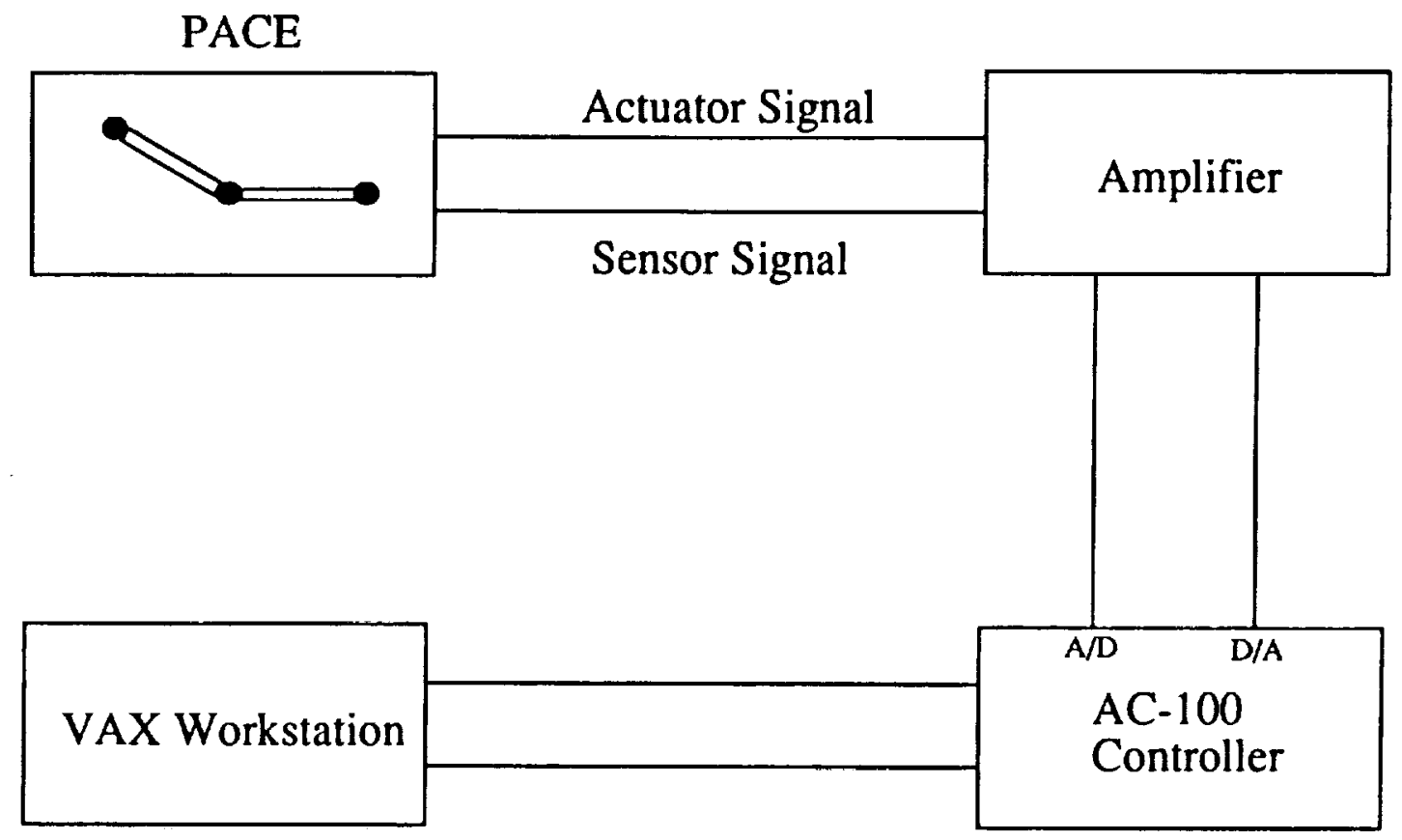

Fig. 2. The Control Loop for the PACE 

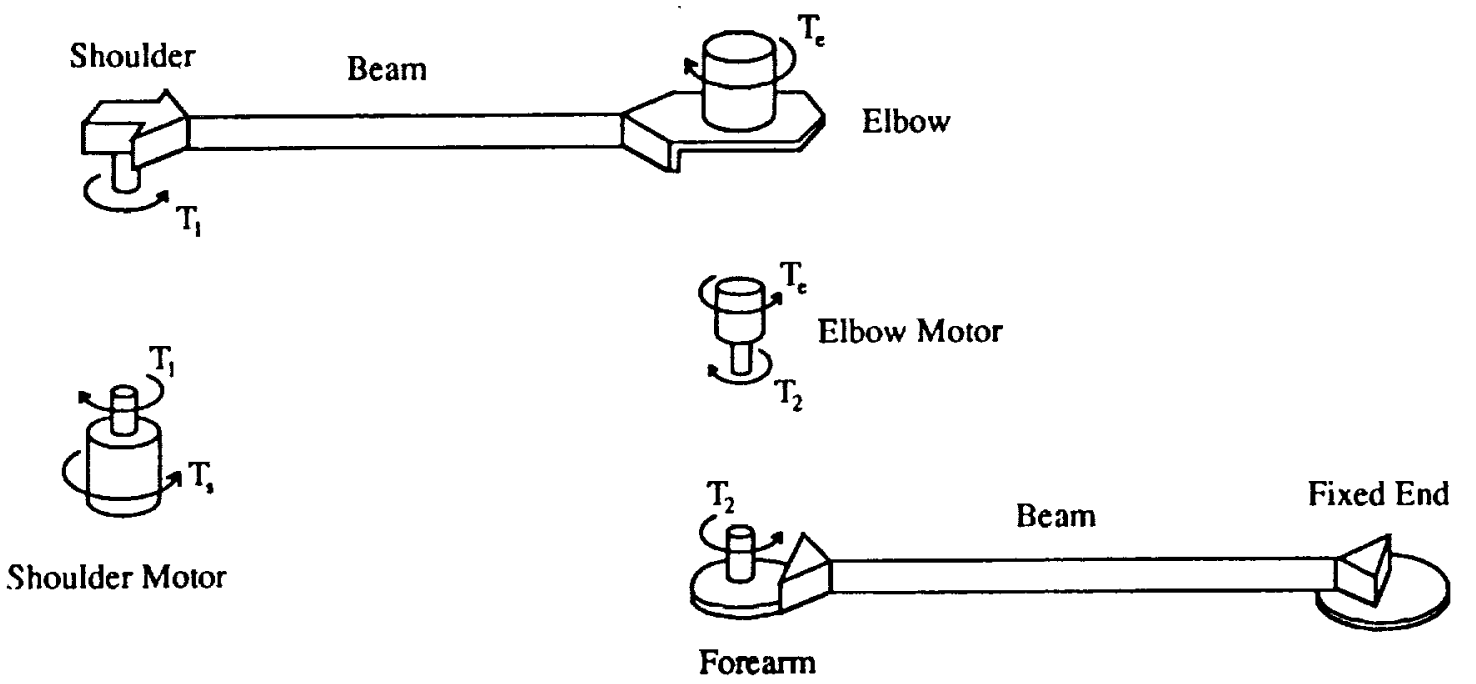

Fig. 3. The Free Body Diagram for the PACE

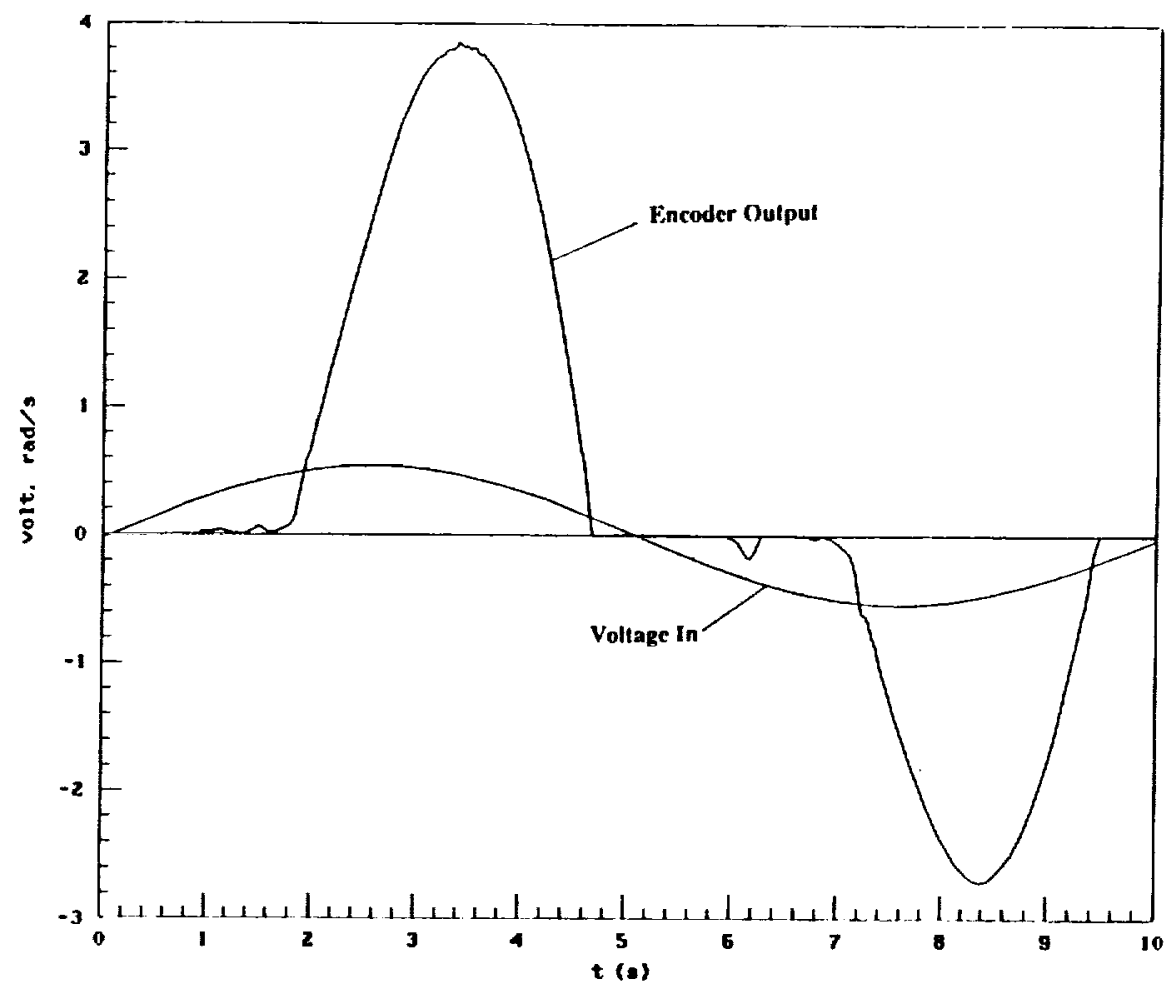

Fig. 4. The Sine-Sweep Test of the Elbow Motor 

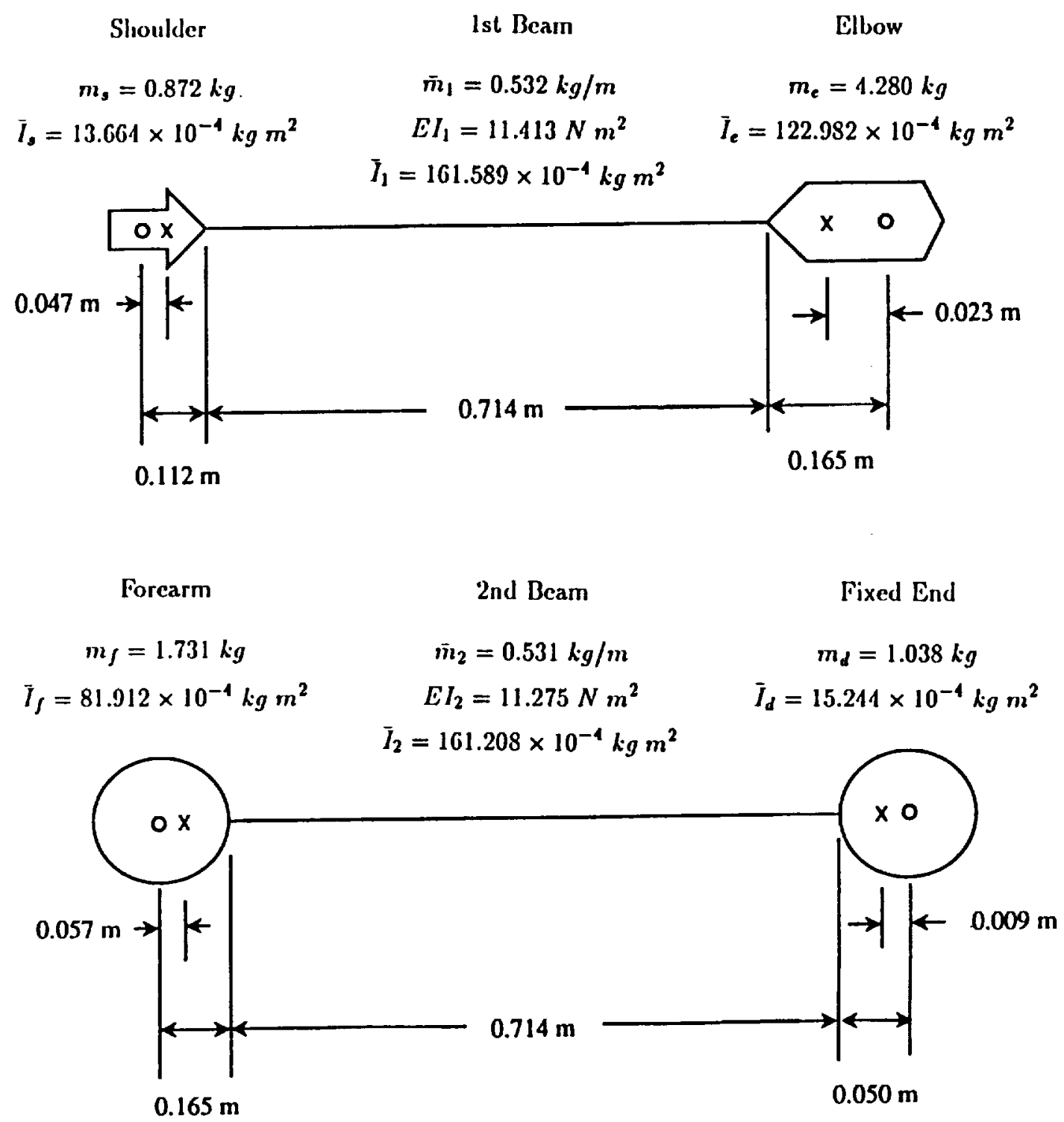

Fig. 5. The Material Properties for the PACE 
